Article

\title{
From Two- to Three-Dimensional Model of Heat Flow in Edge-Emitting Laser: Theory, Experiment and Numerical Tools
}

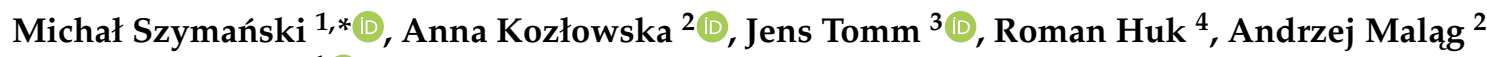 \\ and Marian Rusek ${ }^{1}$ (D) \\ 1 Institute of Information Technology, Warsaw University of Life Sciences-SGGW, ul. Nowoursynowska 159, \\ 02-776 Warsaw, Poland; marian_rusek@sggw.edu.pl \\ 2 Łukasiewicz Research Network-Institute of Microelectronics and Photonics, Aleja Lotników 32/46, \\ 02-668 Warsaw, Poland; anna.kozlowska@imif.lukasiewicz.gov.pl (A.K.); andrzejmalag635@gmail.com (A.M.) \\ 3 Max-Born-Institut, Max-Born-Str. 2 A, 12489 Berlin, Germany; tomm@mbi-berlin.de \\ 4 Science and Technology Park Poland-East in Suwałki Ltd., ul. Innowacyjna 1, 16-400 Suwałki, Poland; \\ roman@huk.waw.pl \\ * Correspondence: michal_szymanski@sggw.edu.pl
}

check for updates

Citation: Szymański, M.; Kozłowska, A.; Tomm, J.; Huk, R.; Malag, A.; Rusek, M. From Two- to

Three-Dimensional Model of Heat Flow in Edge-Emitting Laser: Theory, Experiment and Numerical Tools. Energies 2021, 14, 7006. https:// doi.org/10.3390/en14217006

Academic Editors: Lubomir Bena, Damian Mazur and Bogdan Kwiatkowski

Received: 4 September 2021

Accepted: 21 October 2021

Published: 26 October 2021

Publisher's Note: MDPI stays neutral with regard to jurisdictional claims in published maps and institutional affiliations.

Copyright: (c) 2021 by the authors. Licensee MDPI, Basel, Switzerland. This article is an open access article distributed under the terms and conditions of the Creative Commons Attribution (CC BY) license (https:/ / creativecommons.org/licenses/by/ $4.0 /)$.

\begin{abstract}
Mathematical modeling of thermal behavior of edge-emitting lasers requires the usage of sophisticated time-consuming numerical methods like FEM (Finite Element Method) or very complicated 3D analytical approaches. In this work, we present an approach, which is based on a relatively simple 2D analytical solution of heat conduction equation. Our method enables extremely fast calculation of two crucial physical quantities; namely, junction and mirror temperature. As an example subject of research, we chose self-made $p$-side-down mounted InGaAs/GaAs/AlGaAs laser. Purpose-designed axial heat source function was introduced to take into account various mirror heating mechanisms, namely, surface recombination, reabsorption of radiation, Joule, and bulk heating. Our theoretical investigations were accompanied by experiments. We used micro-Raman spectroscopy for measuring the temperature of the laser front facet. We show excellent convergence of calculated and experimental results. In addition, we present links to freely available self-written Matlab functions, and we give some hints on how to use them for thermal analysis of laser bars or quantum cascade lasers.
\end{abstract}

Keywords: catastrophic optical damage; edge-emitting laser; heat conduction equation; mirror temperature; temperature distribution; thermal analysis

\section{Introduction}

One can safely say that the demand for high-power edge-emitting lasers will not expire in foreseeable future. At least two reasons for this can easily be mentioned. Firstly, these devices are widely used in many applications such as solid-state laser pumping, telecommunication, medicine, material processing, and 3D sensing [1,2]. Secondly, dynamic development of epitaxial or processing techniques enables constant progress in obtaining devices with better and better parameters. Multilayer semiconductor structures of precisely selected thicknesses and various chemical compositions, including the wide class of quantum cascade lasers (QCL's) [3], allow for generation of wavelengths from a very wide range. Smart modifications of geometry, like contact tapering, lead to improvement in beam quality and increasing efficiency [4]. Long resonators (of course compared to surface-emitting lasers) enable effective usage of quantum dots, which usually are sparsely distributed throughout the heterostructure because of crystalline growth conditions [5].

It is believed that edge-emitting lasers are prized mainly due to their high output powers. There is much truth in it, and this highlights a certain flaw of these devices, namely, thermal management problems. Radiative processes necessary for light generation are accompanied by undesired heat-generating processes, like nonradiative recombination, 
Auger recombination, Joule effect, or surface recombination. Even for highly efficient laser sources, a great amount of energy supplied by pumping current is converted into heat. Thus, detailed insight into thermal effects in the edge-emitting lasers is the essential condition of obtaining the improved devices [6].

The rising temperature of the laser has a negative effect on its performance: the threshold current increases, the spectral characteristics shift, and the power conversion efficiency decreases. The most destructive effects are observed at high pumping levels. The milder of these is reversible and is referred to as a thermal roll-over: the user increases the pumping current, and at the same time, observes the decrease in optical power (instead of the expected increase). The catastrophic optical mirror damage (COMD), on the other hand, is the worst variant, as it irreversibly destroys the device by melting the mirrors.

The problems described above resulted in numerous researches in the field of thermal analysis of semiconductor lasers. Refs. [7,8] can be given as examples of one of the earliest and recent reports, respectively. The unwavering interest in this subject can be explained by expectations of obtaining greater and greater optical powers. Thus, the researchers design very sophisticated techniques of facet temperature reduction $[9,10]$, investigate degradation mechanisms [11] or look for more efficient cooling systems [12].

At low pumping levels thermal behavior of an edge-emitting laser can be described by the $2 \mathrm{D}$ stationary heat conduction equation $[7,13,14]$. However, the complete thermal picture, including the region close to the mirrors and suitable for high pumping levels, requires very complicated 3D analytical models [15-17] or usage of sophisticated numerical methods for solving partial differential equations [18-20]. In Ref. [21] we elaborated an original theoretical concept allowing for calculation junction as well as mirror temperatures, and at the same time, avoid the drawbacks mentioned above. We revisited a relatively simple analytical, 2D stationary model of heat flow in an edge-emitting laser. We showed that it could effectively describe a 3D case if used two times adequately.

In this paper, we substantially develop our concept by precise determination of heating function versus axial direction. The essential part of our work is the quantitative currentdependent model of mirror heating. It emerges from the formula for heating caused by surface recombination proposed in [16] and-according to thorough experimental investigations [22]—is extended to include other vital mechanisms, like reabsorption of radiation, Joule heating, or bulk heating. Our theoretical investigations are accompanied by facet temperature measurements in continuous-wave mode performed by micro-Raman spectroscopy. We show excellent convergence between experimental and calculated values of temperature presented in this paper. In addition, we give links to freely available Matlab functions we developed and used in our investigations and formulate some hints on how to use them in various cases, like the assumption of a real or ideal heat sink or a quantum cascade laser.

This paper is organized as follows. In Section 2 our model of heat flow in an edge-emitting laser is presented with particular emphasis on heating in axial direction (Section 2.3). In Section 3 an example device and its parameters are presented. In Section 4 we describe the experimental setup. The results are presented in Section 5 and discussed in Section 6. We finish with some conclusions in Section 7.

\section{The Thermal Model}

Our original concept and related mathematical derivations can be found in [21]. The most important parts of this work are briefly recalled in Sections 2.1 and 2.2, while Section 2.3 is extensive since it contains essential addition, namely, a recipe for calculating current-dependent heating function in axial direction.

\subsection{Basic 2D Model}

To formulate the basic thermal model, we focus on parallel cross-section (plane $x-y$ ) depicted in Figure 1 and let $L \rightarrow \infty$. In this way, our laser becomes a rectangular rod composed of various semiconductor layers. Each layer is characterized by its thickness 
and thermal conductivity $\lambda$. The 2D stationary heat conduction equation and boundary conditions for such a rod can be written as:

$$
\begin{gathered}
\nabla(\lambda(y) \nabla T(x, y))=-g(x), \\
T\left(x, y_{b}\right)=0, \\
\frac{\partial}{\partial x} T\left( \pm \frac{b}{2}, y\right)=0, \\
-\lambda_{2 K} \frac{\partial}{\partial y} T\left(x, y_{t}\right)=\alpha T\left(x, y_{t}\right),
\end{gathered}
$$

where $T$ is the temperature exceeding the ambient temperature conventionally assumed to be zero, and $\alpha$ is the heat transfer coefficient.

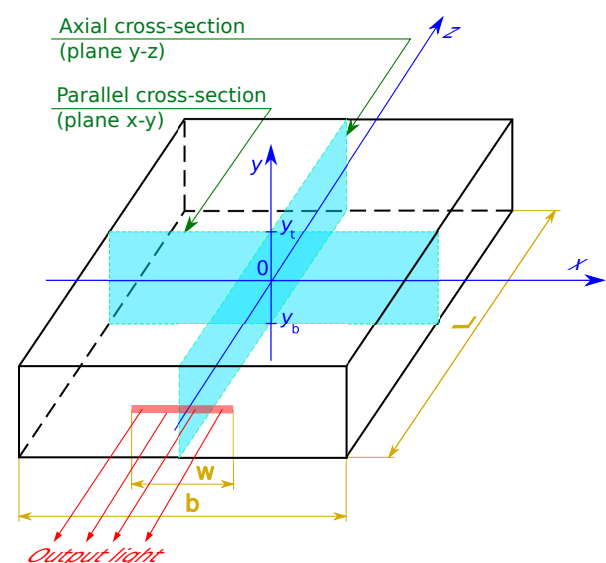

Figure 1. Schematic view of the laser chip. Symbols $w, b, L, y_{t}$ and $y_{b}$ denote active layer width, structure width, cavity length, and the coordinate of top and bottom surface, respectively. Note that at the edges of both cross-sections, the same boundary conditions are assumed.

In our approach, the heat is generated only by an infinitely thin stripe placed between layer No 1 and 2. Thus, we introduce function $g(x)$ as in Figure 2, where

$$
J=\frac{U I-P_{\text {out }}}{w L},
$$

$I$ is the pumping current, $U$-voltage and $P_{\text {out }}$-optical power. Since $g(x)$ is even, we can expand it into a Fourier cosine series:

$$
g(x)=a_{0}+\sum_{k=1}^{\infty} a_{k} \cos \left(k \omega_{0} x\right),
$$

and, in our further considerations, the heat source is represented by coefficients

$$
a_{0}=\frac{J w}{b}, \quad a_{k}=\frac{2 J}{k \pi} \sin \left(\frac{k \pi w}{b}\right) .
$$

Note that $y_{b}$ indicates the bottom of the laser chip if one assumes the ideal heat sink $\left(\lambda_{H S} \rightarrow \infty\right)$. Taking into account the nonideal heat sink (finite $\lambda_{H S}$ ) requires increasing the dimensions $y_{b}$ and $b$ and was thoroughly explained in Ref. [14]. 
The separation-of-variables approach provides the solution in a two-fold form. In the layers above the active layer ( $n$ - even), it is described by

$$
\begin{aligned}
T_{n}(x, y)= & A_{2 K}^{(0)}\left(w_{A, n}^{(0)}+w_{B, n}^{(0)} y\right)+ \\
& \sum_{k=1}^{\infty} A_{2 K}^{(k)}\left[w_{A, n}^{(k)} \exp \left(\mu_{k} y\right)+w_{B, n}^{(k)} \exp \left(-\mu_{k} y\right)\right] \cos \left(\mu_{k} x\right)
\end{aligned}
$$

while under the active layer ( $n$-odd), it takes the form:

$$
\begin{aligned}
T_{n}(x, y)= & A_{2 K}^{(0)} \frac{w_{A, 2}^{(0)}}{w_{A, 1}^{(0)}}\left(w_{A, n}^{(0)}+w_{B, n}^{(0)} y\right)+ \\
& \sum_{k=1}^{\infty} A_{2 K}^{(k)} \frac{w_{A, 2}^{(k)}+w_{B, 2}^{(k)}}{w_{A, 1}^{(k)}+w_{B, 1}^{(k)}}\left[w_{A, n}^{(k)} \exp \left(\mu_{k} y\right)+w_{B, n}^{(k)} \exp \left(-\mu_{k} y\right)\right] \cos \left(\mu_{k} x\right) .
\end{aligned}
$$

In (8) and (9) $k$ numerates so called heat modes and $\mu_{k}=2 k \pi / b$. The derivations of coefficients $w_{A, n}^{(k)}, w_{B, n}^{(k)}$ and $A_{2 K}^{(k)}$ can be found in [14]. Here, we present the final formulas only for the last one distinguishing cases $k=0$ and $k \geqslant 1$ :

$$
\begin{gathered}
A_{2 K}^{(0)}=\frac{a_{0}}{\lambda_{1} \frac{w_{A, 2}^{(0)} w_{B, 1}^{(0)}}{w_{A, 1}^{(0)}}-\lambda_{2} w_{B, 2}^{(0)}}, \\
A_{2 K}^{(k)}=\frac{a_{k}}{\mu_{k}\left[\lambda_{1} \frac{w_{A, 2}^{(k)}+w_{B, 2}^{(k)}}{w_{A, 1}^{(k)}+w_{B, 1}^{(k)}}\left(w_{A, 1}^{(k)}-w_{B, 1}^{(k)}\right)-\lambda_{2}\left(w_{A, 2}^{(k)}-w_{B, 2}^{(k)}\right)\right]} .
\end{gathered}
$$

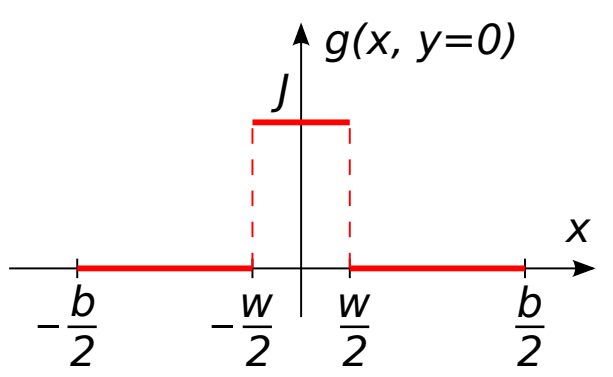

Figure 2. Function describing heating in lateral direction. $y=0$ is plane of active layer.

\subsection{D Thermal Model Applied Twice in Different Planes}

In numerous works it was shown that side walls and mirrors of an edge-emitting laser can be treated as thermally isolated surfaces $[8,17,19,20]$. Such an assumption is also used here, so the boundary conditions (2)-(4) take the form:

$$
T\left(x, y_{b}\right)=0, \quad \frac{\partial}{\partial x} T\left( \pm \frac{b}{2}, y\right)=0, \quad \frac{\partial}{\partial y} T\left(x, y_{t}\right)=0
$$

after substituting $\alpha=0$. Consequently, we find that the thermal model described in Section 2.1 can be easily adapted for predicting the temperature along the $z$ direction. Looking at the axial cross-section of the investigated device (Figure 1), we see that the structure geometry is the same and boundary conditions exactly suit to the new situation, since thermally insulated side walls are replaced by thermally insulated mirrors. The only modification concerns the heat source function. The problem is complex and will be explained with details in the next section. Here, let's only assume that $g(y=0, z)$ is a 
symmetrical function as presented in Figure 3. Such a function can also be expanded into Fourier series (6) and coefficients take the form:

$$
\left.a_{0}=\frac{2}{L}\left[g_{a}\left(L-2 d_{\text {sur }}\right)+2 d_{\text {sur }} g_{m}\right)\right], \quad a_{k}=\frac{2\left(g_{a}-g_{m}\right)}{n \pi} \sin \left[\frac{2 n \pi}{L}\left(\frac{L}{2}-d_{\text {sur }}\right)\right] .
$$

Now $T(z, y)$ can be calculated using the model from section 2.1 replacing $x$ by $z$ and coefficients (7) by (13). In further text, we use symbols $T_{m}=T(z=-L / 2, y=0)$ for mirror temperature and $T_{j}=T(z=0, y=0)$ for junction temperature.

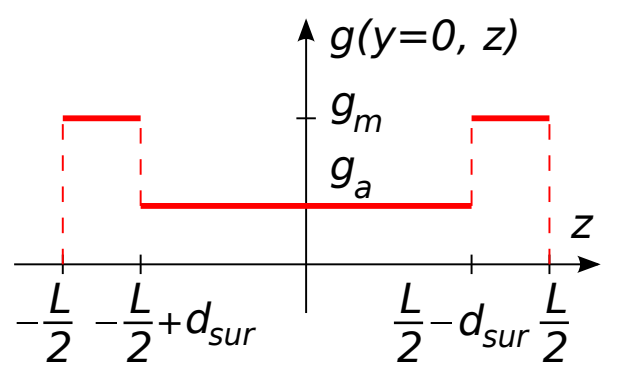

Figure 3. Function describing heating in axial direction. $d_{\text {sur }}$ determines thickness of extra heated region near mirror surface.

Finding values $g_{m}$ and $g_{a}$ is preceded by assuming uniform heating $g_{m}=g_{a}=J_{e f f}$ and applying the bisection method for calculating the value $J_{e f f}$ for which

$$
T(z=0, y=0)=T(x=0, y=0) .
$$

Physically, it means that our thermal model must provide the same junction temperature no matter which plane $(x-y$ or $y-z)$ is considered. The quantity $J_{e f f} w L$ is the power dissipated in the entire resonator and must be equal to the sum of power dissipated near the mirrors (regions $-L / 2<z<-(L / 2)+d_{\text {sur }}$ and $(L / 2)-d_{\text {sur }}<z<L / 2$ ) and deep inside the resonator (region $-(L / 2)+d_{\text {sur }}<z<(L / 2)-d_{\text {sur }}$ ):

$$
J_{e f f} w L=2 g_{m} w d_{s u r}+g_{a} w\left(L-2 d_{s u r}\right) .
$$

Below, we relate heating to pumping current $\left(g_{m} \rightarrow g_{m}(I), g_{a} \rightarrow g_{a}(I)\right)$, and thus determine precisely the function $g(y=0, z)$ from Figure 3 .

\subsection{Heating in Axial Direction}

The value of $g_{m}$ can be assessed by combining the results of researches reported in $[16,22]$. Thorough experimental investigations in the latter work allowed for drawing functional relationships between mirror heating and pumping current. Originally both physical quantities are expressed in arbitrary units (see Figure 1 in [22]). In the further part of our work, they are successively transformed into $\mathrm{W} / \mathrm{m}^{2}$ and Amperes, respectively. Plus, we reject the range below the threshold $\left(I<I_{t h}\right)$ as uninteresting from the thermal point of view.

The original experimental diagram is retrieved by using the linear approximations. The functional form can be written as:

$$
h=b_{s r}+\left(a\left(I-I_{\text {shift }}\right)+b\right),
$$

where constant term $b_{s r}$ pertaining to surface recombination is extracted since it will be useful in further calculations. $I_{\text {shift }}$ was introduced to transform unity of current from arbitrary to Amperes while considering real laser. Phenomenological heating contribution proportional to the injection current is included [22]. The resultant diagram is presented in Figure 4. 
In [16], we find expression for mirror heating induced by surface recombination above threshold $\left(I \geq I_{\text {th }}\right)$ :

$$
q=\hbar \omega v_{0} \frac{m}{m+v_{0} / D} N_{t h}
$$

where $\hbar$ is the Planck's constant, $e$-electron charge, $V$-volume of the active layer, $\omega$-angular frequency, $D$-carrier diffusivity, $v_{0}$ — surface recombination velocity, $I_{t h}-$ threshold current, $N_{t h}$-threshold carrier density and

$$
m=\sqrt{\frac{(1 / \tau)+A P_{t}}{D}} .
$$

The photon density inside the laser resonator $P_{t}$ is related to $P_{\text {out }}$ through

$$
P_{t}=\frac{(1+R) \Gamma P_{o u t}}{w y_{0}(1-R) \hbar \omega v_{g}}
$$

where $v_{g}$ is the photon group velocity, $\tau$-carrier lifetime, $A$-the gain, $y_{0}$-active layer thickness, $R$-front facet reflectivity, $\Gamma$ - the optical confinement factor and $\hbar \omega$ - the photon energy.

It is important to note that in [16], the heat source is a rectangle stuck to the mirror and active layer joint. In our model, heat source is also a rectangle, but it is also a segment of an infinitely thin active layer. Thus, $q$ must be rescaled:

$$
q_{\text {res }}=\frac{y_{0}}{d_{\text {sur }}} q \text {. }
$$

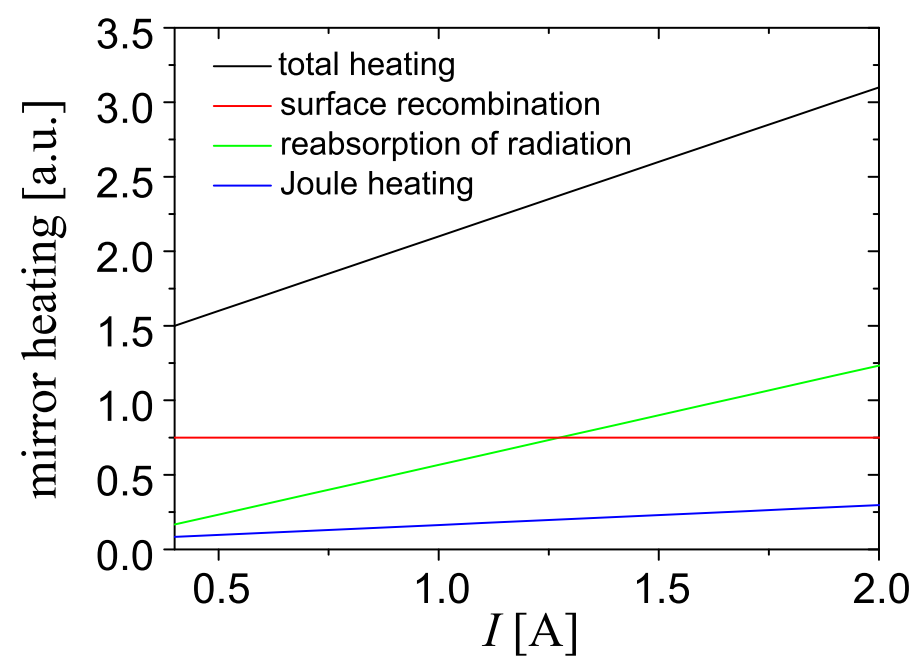

Figure 4. Mirror heating vs pumping current. Color lines show contributions caused by various phenomena [22].

Note that current-dependent quantity $b_{s r} / h$ determines the dimensionless contribution of surface recombination to the total mirror heating. Thus, the total mirror heating expressed in $\mathrm{W} / \mathrm{m}^{2}$ can be calculated as

$$
g_{m}=\frac{h}{b_{s r}} q_{r e s} .
$$

Now, using Equation (15), we can calculate

$$
g_{a}=\frac{J_{e f f} L-2 g_{m} d_{s u r}}{L-2 d_{\text {sur }}}
$$


and define parameter

$$
p=\frac{2 q_{r e s} d_{\text {sur }}}{J_{\text {eff }} L},
$$

which determines the amount of the total effective power released in the vicinity of mirrors.

\section{Description of the Investigated Device}

For our thermal analysis we choose a $p$-side-down mounted edge-emitting InGaAs / GaAs/AlGaAs laser. The heterostructure is described layer by layer in Table 1. Heat sink is taken into account. Figure 5 shows the light-current and voltage-current characteristics measured for $0<I<5 I_{t h}$.

Table 1. Layer parameters used in the thermal model. Note that the active layer thickness was neglected, i.e., $y_{0} \rightarrow 0$ according to $[7,14]$.

\begin{tabular}{cccc}
\hline Layer Number & Description & $\begin{array}{c}\text { Thickness } \\
{[\mu \mathrm{m}]}\end{array}$ & $\begin{array}{c}\text { Thermal Conductivity } \\
{[\mathbf{W} /(\mathbf{m} \text { K)] }}\end{array}$ \\
\hline 14 & GaAs substrate & 100 & 55.0 \\
12 & GaAs buffer & 0.5 & 55.0 \\
10 & AlGaAs n-cladding & 3.5 & 11.18 \\
8 & AlGaAs gradient & 0.05 & 9.69 (averaged) \\
6 & AlGaAs barrier & 0.05 & 11.0 \\
4 & AlGaAs waveguide & 0.18 & 13.72 \\
2 & GaAs spacer & 0.005 & 55.0 \\
- & InGaAs QW active layer & 0 & - \\
1 & GaAs spacer & 0.005 & 55.0 \\
3 & AlGaAs waveguide & 0.18 & 13.72 \\
5 & AlGaAs barrier & 0.05 & 11.00 \\
7 & AlGaAs gradient & 0.05 & 9.69 (averaged) \\
9 & AlGaAs p-cladding & 3.5 & 11.18 \\
11 & GaAs contact layer & 0.3 & 55.0 \\
13 & Cu heat sink & 2000 & 384.0 \\
\hline
\end{tabular}

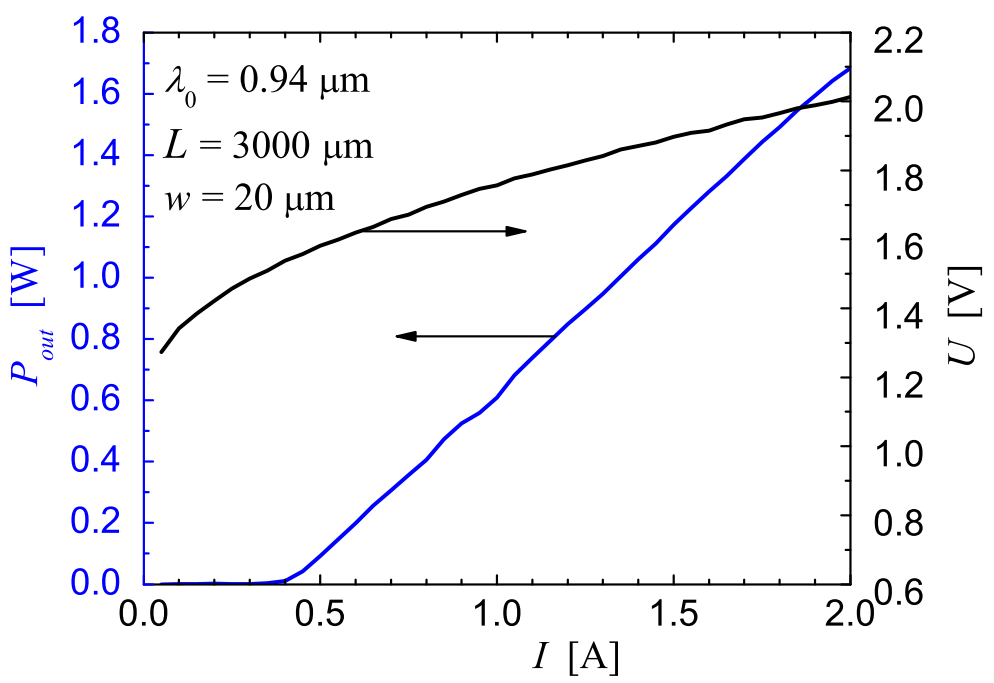

Figure 5. Characteristics and selected parameters of investigated device.

\section{Experimental Setup}

Our facet temperature measurements in the continuous-wave mode were performed by micro-Raman spectroscopy (see Figure 6). An SI TriVista TR 557 Raman spectrometer, equipped with a microscope and a liquid nitrogen-cooled CCD camera, monitors the Stokes GaAs-like TO phonon line $\left(\simeq 267 \mathrm{~cm}^{-1}\right)$ generated in the $\mathrm{Al}_{0.18} \mathrm{Ga}_{0.82}$ As waveguide. As an 
excitation source, the $442 \mathrm{~nm}$ laser line of a He-Cd laser Kimmon IK57511-G is used. Focused by a $100 \times$ objective, a surface temperature probe with an information depth $<100 \mathrm{~nm}$ and lateral extension $<1 \mu \mathrm{m}$ is created. Typical measurement times for a single spectrum (one temperature data point) are in the order of 100 s. Simultaneously, the facet surface and the laser emission are observed. In this way, changes in the facet or a decrease in the emission power could be detected. All continuous-wave measurements were performed at stabilized heat sink temperatures of $T_{h s}=(25.0 \pm 0.2){ }^{\circ} \mathrm{C}$. Temperature calibration is based on measurements with devices intentionally set to certain elevated temperatures.
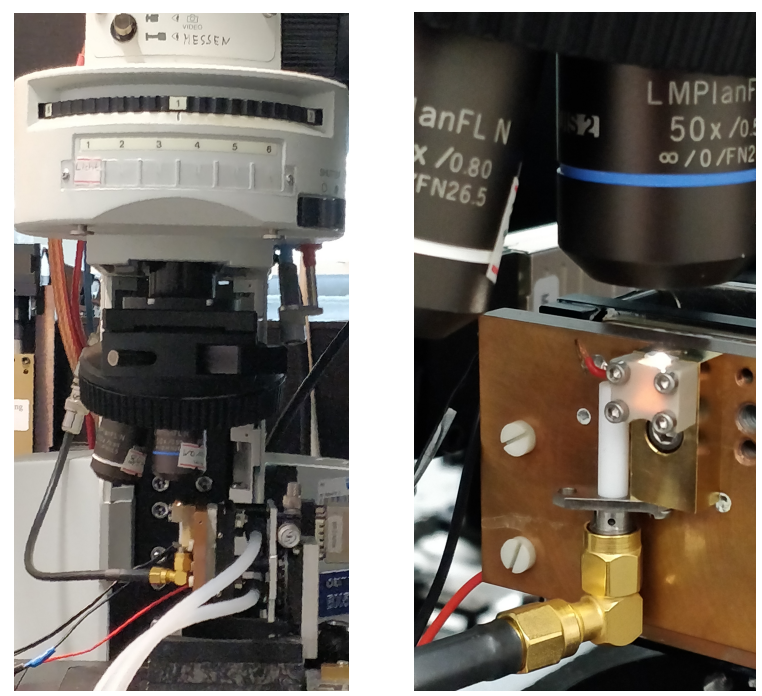

Figure 6. Photos of our experimental set. On the left, the microscope with thermoelectric temperature control is shown. Black and red cables power the Peltier element, while white hoses supply water for cooling. The operating current is supplied by the thick black cable through the SMA R/A adapter. On the right, the enlarged fragment with mounted device is shown. The front facet of the diode laser is at the bright white spot in the center. The illumination comes from the microscope objective.

\section{Results}

The light-current and voltage-current characteristics from Figure 5 provide us data: $I, U$ and $P_{\text {out }}$. $I_{\text {th }}=0.4 \mathrm{~A}$, so $I_{\text {shift }}=0.6 \mathrm{~A}$. Since in [23] the carrier diffusion length in InGaAs/GaAs quantum well heterostructures is reported to be $2.7 \mu \mathrm{m}$, this is the value assumed for $d_{\text {sur }}$. Following material data presented in [16], we set $v_{0}=4000 \mathrm{~m} / \mathrm{s}$, $D=9.6 \cdot 10^{-4} \mathrm{~m}^{2} / \mathrm{s}, \tau=4 \cdot 10^{-9} \mathrm{~s}$ and $A=2 \cdot 10^{-12} \mathrm{~m}^{3} / \mathrm{s}$. Additionally, for our structure $y_{0}=7 \mathrm{~nm}$ (this value is used for calculation of $g_{m}$ and neglected while temperature calculations-see Table 1 ) and $\Gamma=0.0065, R=0.1, v_{g}=3 \cdot 10^{8} / n_{\text {eff }}[\mathrm{m} / \mathrm{s}], n_{\text {eff }}=3$ are parameters calculated by commercial software [21]. To obtain the most reliable values of $T$, we decide to take into account the nonideal heat sink. For this purpose, we include the copper layer (layer number 13 in Table 1) with its actual thickness $2000 \mu \mathrm{m}$ and assume $b=5000 \mu \mathrm{m}$, which is 10-times larger than true structure width. Such a procedure is explained thoroughly in our previous work [14].

Figure 7 shows temperature profiles calculated by our Matlab software. When analyzing graphs, keep in mind that $y_{t}=104.285 \mu \mathrm{m}, y=-4.085 \mu \mathrm{m}$ is the contact plane of the structure and the heat sink, $y_{b}=-2004.085 \mu \mathrm{m}$ and the device is symmetrical with respect to the plane $x=0$. Thus, the 2D color map is drawn for $x>0$ only and covers the chip area together with a piece of the heat sink. Looking at the transverse temperature profile (upper-right), we see that the boundary conditions (2) and (4) are fulfilled, because $T$ starts from zero (i.e., ambient temperature following the convention assumed in Section 2.1) at the bottom of the heat sink and reaches the upper contact horizontally, which indicates no heat transfer through this plane. Analogically, the lateral profile (lower left) shows the flattening of $T$ towards increasing $x$ values, which is a consequence of the boundary condition (3). The essential output, namely the axial temperature profile, is shown lower 
right. From this chart, we can extract the most important results of our thermal analysis, i.e., junction and mirror temperatures. Calculation of $T_{j}$ was already the subject of our detailed research in the work [14]. We presented there the experiments that proved the correctness of the analytical model described in Section 2.1. Therefore in this work, we mainly focus on issues related to mirror temperature.
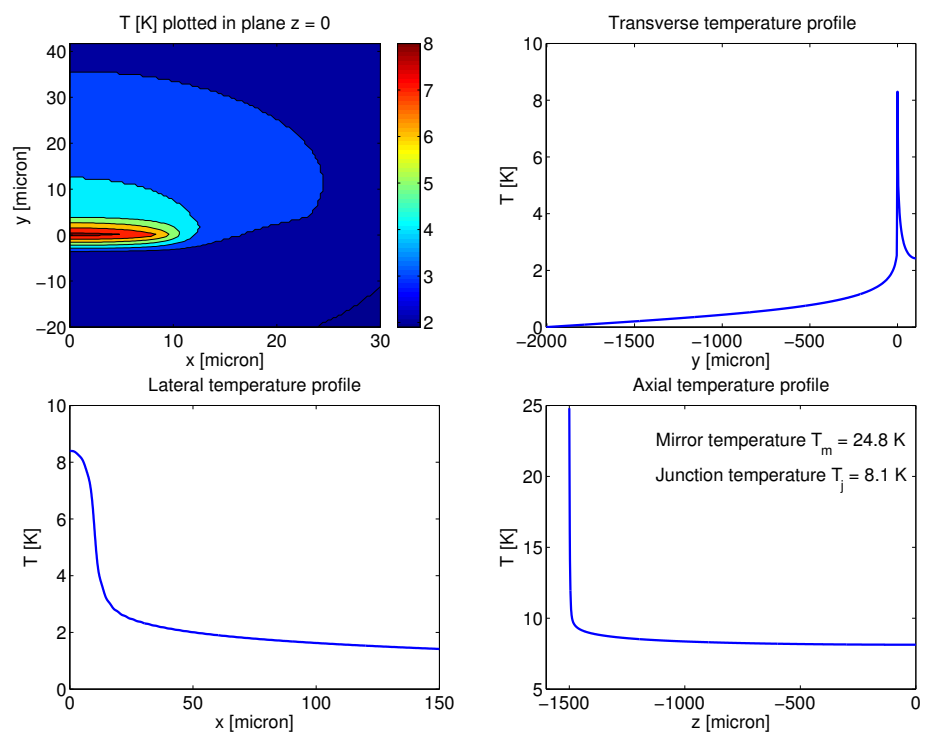

Figure 7. Graphical output of our Matlab software. Note that $x, y, z$ are positive or negative according to the orientation of the coordinate system presented in Figure 1.

Comparison of our micro-Raman spectroscopic experiment with the model is presented in Figure 8. The calculated profile $T_{m}(I)$ is within the measurement error area, which should be considered as an evident success, especially that, in thermal modeling, the values of parameters are burdened by errors difficult to quantify. For example, thermal contact between chip and heat sink may be affected by voids in the solder, the laser structure may suffer from an overhang, $v_{0}$ depends on mirror passivation, and $d_{\text {sur }}$ may differ for different heterostructures.

For further discussion, it is reasonable to define

$$
\Delta T=\left|T_{m}^{(\text {calc })}-T_{m}^{(\text {meas })}\right|
$$

as the deviation of the calculated values from the measured ones. Analysing data from Figure 8, we find $0.1<\Delta T<3.9 \mathrm{~K}$. Now, keeping in mind the problems with the exact determination of the parameter values, we decided to investigate the influence of parameter inaccuracies for thermal analysis. The results are presented in Figures 9 and 10. 


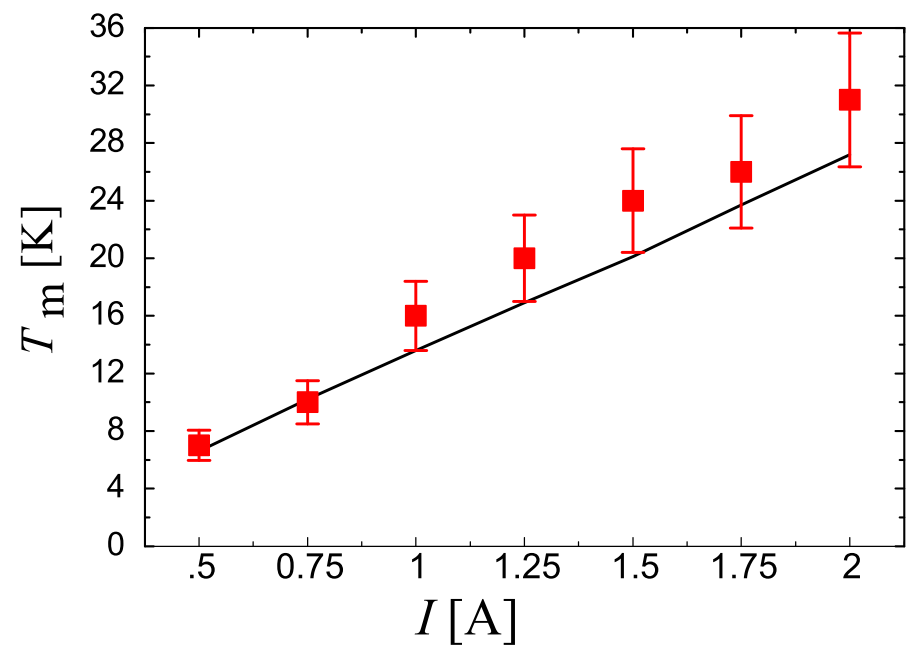

Figure 8. Mirror temperature calculated (solid line) and measured by micro-Raman spectroscopy (red points with error bars) versus pumping current. Note that zero on vertical axis represents ambient temperature, and horizontal axis begins with threshold current $I_{t h}=0.4 \mathrm{~A}$.

Experimental studies of surface recombination performed on a wide variety of candidate materials for nanostructure light-emitting diodes provide ranges of $v_{0}$ rather than specific values $[24,25]$. Therefore, we plotted $\Delta T$ versus pumping current for several $v_{0}$ 's (Figure 9). Increasing $v_{0}$ leads to the reduction of the maximum value of $\Delta T$, which suggests that the influence of surface recombination in the case of our specific device was underestimated.

Carrier diffusion length for different material constellations and specifically processed devices is another parameter that eludes precise quantitative description. Figure 10 shows that $d_{\text {sur }}$ shrinked to $1 \mu \mathrm{m}$ leads to slight decrease of $\max (\Delta T)$.

The plots in Figures 9 and 10 are not monotonic. However, in view of $15 \%$ error of $T_{m}^{(\text {meas })}$, we do not analyze in detail their shapes. Instead, we formulate conclusions based mainly on $\max (\Delta T)$.

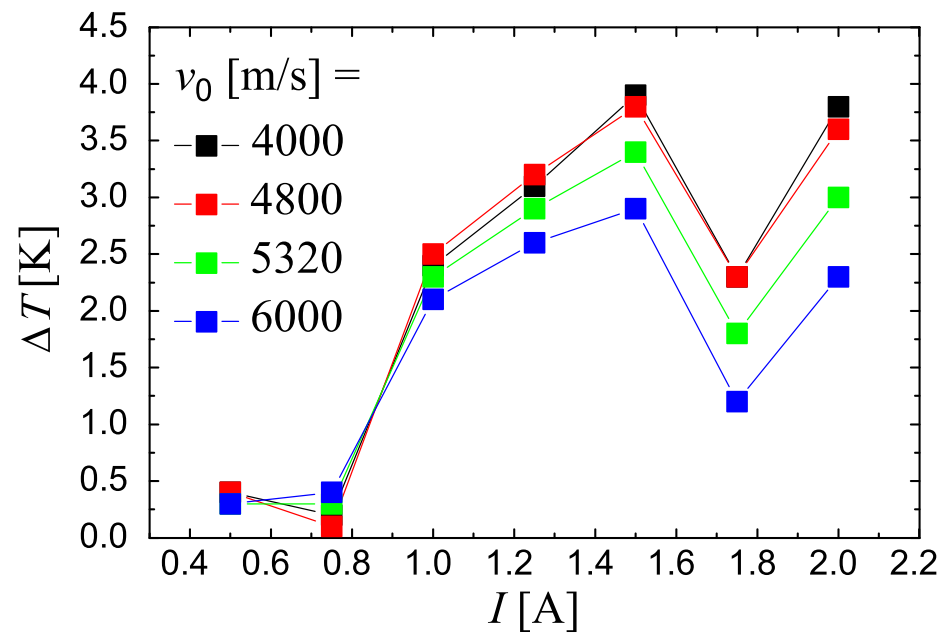

Figure 9. Influence of inaccuracies of determining $v_{0}$. Lines connecting points are drawn for readability only. Note that $\max (\Delta T)$ decreases with increasing $v_{0}$. 


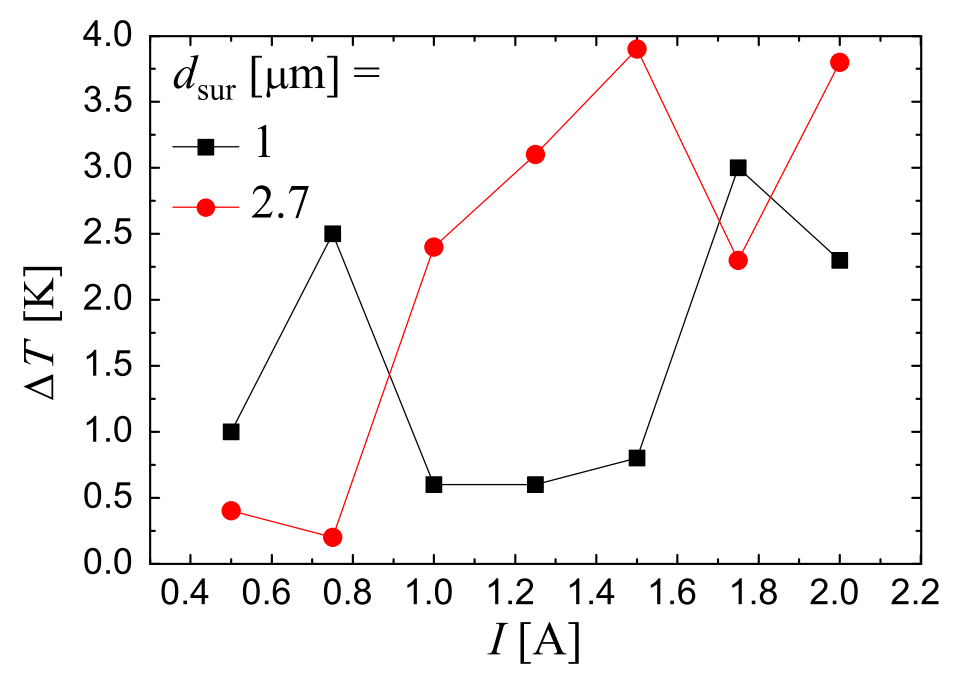

Figure 10. Influence of inaccuracies of determining $d_{\text {sur }}$. Lines connecting the points are drawn for readability only. Note that reducing $d_{\text {sur }}$ resulted in reduction of $\max (\Delta T)$.

\section{Discussion}

We showed that a relatively simple $2 \mathrm{D}$ analytical thermal model could be successfully adapted for analysis of 3Dheat flow in an edge-emitting laser. The essence of our concept relies on using 2D expressions for $T$ twice: in the plane $x-y$, and next in the plane $y-z$ (see Figure 1). The procedure is possible because at the edges of both contours, and the same boundary conditions can be assumed.

Thermal management in edge-emitting lasers was the subject of many works. Some authors developed analytical [15-17], while some others developed numerical models [18-20]. In this situation, the need to justify the return to this topic is understandable. It is done below.

Analytical models are created for the areas adjacent to the mirrors only. In Ref. [15] $3 \mathrm{D}$ transient heat conduction equation is considered while modeling the COMD process. The solution is valid only for the fast thermal runaway process because of the approximations involved. The most similar approach to ours can be found in [16], where separation-of-variables method was used for solving 3D stationary heat conduction equation with a rectangular source function releasing heat $q$ induced by surface recombination (see Equation (17)). However, the authors-looking for the necessary simplificationsneglected other important heat-generating mechanisms, like reabsorption of radiation or Joule heating.

Numerical models turned out to be more versatile compared to their analytical counterparts. They allowed for taking into account various heating mechanisms and successfully provided temperatures for entire devices. However, the problem with computational time arose. The authors of [20] reported that the full 3D simulation took $5 \mathrm{~h} 11 \mathrm{~min}$ for a given set of parameters. Such computational effort is not acceptable while optimization conducted with the usage of heuristics algorithms when thousands or more repetitive calculations are required [26]. In contrast, we report that obtaining the textual output $T(z)$ from Figure 7 takes approximately $2.5 \mathrm{~s}$ on a standard laptop.

Numerical models based on FEM are difficult to apply if the sizes of the constituent elements of the investigated device differ significantly. That is the case encountered here. In Refs. [18-20] the heat flow is considered within the chip only. Physically, it means that the authors assume the ideal sink $\left(\lambda_{H S} \rightarrow \infty\right)$, which may lead to overestimation of real temperatures. In contrast, our approach allows taking into account the whole assembly (chip, heat spreader, and heat sink). It can be done by supplementing the rectangular rod from Figure 1 by additional layer and increasing $b$. The problem was discussed thoroughly in [14]. 
Note also that the purpose-designed heat source function (Figure 3) creates several possibilities for researchers. In the case of a bipolar device (like our InGaAs/GaAs/AlGaAs laser), it can incorporate such heating mechanisms as surface recombination, reabsorption of radiation, Joule, and bulk heating. Unipolar device (i.e., quantum cascade laser) is heated mainly due to dissipative quantum transport processes in the active layer [27] and-in the vicinity of output mirror-due to reabsorption of radiation [28]. Thus, $g_{m}$ and $g_{a}$ should be recalculated adequately, but the shape of the function itself may remain unchanged.

Keep in mind that the heat conduction equation is linear, which allows for the summation of temperatures from different heat sources according to the superposition principle. This fact can be useful in cases where heat sources are spatially extended (for example, thick active region of QCL) or localized in different places (laser bars or stacks).

An essential part of our work was the verification of the proposed model. The calculations were carried out for the self-manufactured device, thanks to which we knew precisely many vital parameters, such as layer thicknesses, their chemical compositions, assembly details, etc. Using the micro-Raman spectroscopy, we measured the mirror temperatures for pumping currents in the range of $I_{t h}<I<5 I_{t h}$ and compared them with the calculated values (Figure 8). The observed discrepancies do not exceed $15 \%$, which we consider a very good convergence. This belief is confirmed by additional calculations motivated as follows. Even knowing the structure of a given laser thoroughly, it is difficult to determine some material parameters precisely. Analysis of $\Delta T$ vs $I$ for the (quite likely) faster than expected surface recombination or shorter carrier diffusion length leads to even more accurate results, which are shown in Figures 9 and 10.

Our model can be regarded as a thermal analog of the effective index approximation, according to which a $2 \mathrm{D}$ waveguide is considered as two 1D waveguides rotated by 90 degrees [29]. However, like all approximations, it exhibits its own limitations. Equations (8) and (9) can be applied within the boundaries of the entire resonator if parallel cross-section is considered (see Figure 1 ). In turn, considering the axial cross-section, one can calculate $T(-L / 2<z<0, y \approx 0)$, i.e., only in one half of the resonator (the one adhering to the front mirror) and near the laser axis. Despite these limitations, we claim that our model is satisfactory because it provides junction and mirror temperatures, which are the most important information expected from thermal analysis of edge-emitting laser.

Our complete Matlab code will be published elsewhere and released as an opensource software soon. Nevertheless, its constituent parts are available now at Matlab's File Exchange section. The software is divided into functions that calculate:

(a) $T(x, y)$ in the cross-section of a multilayer rectangular rod with heat source from Figure 2 [30];

(b) $T(z, y)$ in the cross-section of a multilayer rectangular rod with heat source from Figure 3 [31];

(c) heat power density generated due to surface recombination in the vicinity of mirror and described by Equations (17) or (20) [32];

(d) Thermal Boundary Resistance (TBR) at interfaces between two solids [33].

The last function mentioned above was not used in our work because TBRs can be neglected in standard bipolar devices. In turn, the effect plays a crucial role in quantum cascade lasers. These devices contain active regions composed of a vast number of layers, and thus, interfaces, which significantly hinder heat removal (see for example [34-36]). The method of calculation of the cross-plane thermal conductivity of a quantum cascade laser active region can be found in [37]. The numerical tools [30-33] were downloaded 184 times so far.

\section{Conclusions}

Despite of the continuous improvement of edge-emitting laser designs, thermal issues can still play a key role in their proper operation. Therefore, the search for new models allowing to analyze thermal phenomena quickly and with a low computational cost continues. In this paper, such a model allowing to calculate laser junction as well as mirror 
temperature is presented. Excellent agreement with the experiment is shown. In addition, links to freely available self-written Matlab functions together with some hints on how to use them for thermal analysis of laser bars or quantum cascade lasers are presented. In future work, we plan to conduct a comparative analysis for laser structures made of various materials (arsenide, phosphide, and nitride) using our model. Furthermore, we are going to develop user-friendly full Matlab code, release it as an open source software and publish the detailed manual to enable other researchers to use our model for thermal analysis of a wide class of edge-emitting lasers.

Author Contributions: Conceptualization, M.S. methodology, M.S.; software, M.S., R.H.; validation, J.T.; formal analysis, M.S.; investigation, M.S., A.M., J.T.; resources, A.K.; data curation, A.K.; writingoriginal draft preparation, M.S., J.T., R.H.; writing—review and editing, M.R.; supervision, A.M., A.K.; project administration, M.S. All authors have read and agreed to the published version of the manuscript.

Funding: This research received no external funding.

Conflicts of Interest: The authors declare no conflict of interest.

\section{References}

1. Bachmann, F.; Loosen, P.; Poprawe, R. High Power Diode Lasers: Technology and Applications; Springer: New York, NY, USA, 2007; Volume 128.

2. Wong, A. Next-generation 3D sensing applications enabled with edge-emitting laser diodes (Conference Presentation). In Light-Emitting Devices, Materials, and Applications; Kim, J.K., Krames, M.R., Strassburg, M., Eds.; International Society for Optics and Photonics: Bellingham, WA, USA, 2019; Volume 10940.

3. Vitiello, M.S.; Scalari, G.; Williams, B.; De Natale, P. Quantum cascade lasers: 20 years of challenges. Opt. Express 2015, 23, 5167-5182. [CrossRef] [PubMed]

4. Ma, X.; Qu, H.; Qi, A.; Zhou, X.; Ma, P.; Liu, A.; Zheng, W. High power tapered lasers with optimized photonic crystal structure for low divergence and high efficiency. Semicond. Sci. Technol. 2018, 33, 045010. [CrossRef]

5. Norman, J.C.; Jung, D.; Wan, Y.; Bowers, J.E. Perspective: The future of quantum dot photonic integrated circuits. APL Photonics 2018, 3, 030901. [CrossRef]

6. Liu, X.; Zhao, W.; Xiong, L.; Liu, H. Thermal design and management in high power semiconductor laser packaging. In Packaging of High Power Semiconductor Lasers; Springer: New York, NY, USA, 2015; pp. 53-88.

7. Joyce, W.; Dixon, R. Thermal resistance of heterostructure lasers. J. Appl. Phys. 1975, 46, 855-862. [CrossRef]

8. Wu, D.H.; Zah, C.E.; Liu, X. Three-dimensional thermal model of high-power semiconductor lasers. Appl. Opt. 2019, 58, 3892-3901. [CrossRef] [PubMed]

9. Rinner, F.; Rogg, J.; Kelemen, M.; Mikulla, M.; Weimann, G.; Tomm, J.; Thamm, E.; Poprawe, R. Facet temperature reduction by a current blocking layer at the front facets of high-power InGaAs/AlGaAs lasers. J. Appl. Phys. 2003, 93, 1848-1850. [CrossRef]

10. Arslan, S.; Gündoğdu, S.; Demir, A.; Aydınlı, A. Facet Cooling in High-Power InGaAs/AlGaAs Lasers. IEEE Photonics Technol. Lett. 2018, 31, 94-97. [CrossRef]

11. Souto, J.; Pura, J.L.; Jiménez, J. Thermomechanical issues of high power laser diode catastrophic optical damage. J. Phys. D Appl. Phys. 2019, 52, 343002. [CrossRef]

12. Deng, Z.; Shen, J.; Gong, W.; Dai, W.; Gong, M. Temperature distribution and thermal resistance analysis of high-power laser diode arrays. Int. J. Heat Mass Transf. 2019, 134, 41-50. [CrossRef]

13. Bärwolff, A.; Puchert, R.; Enders, P.; Menzel, U.; Ackermann, D. Analysis of thermal behaviour of high power semiconductor laser arrays by means of the finite element method (FEM). J. Therm. Anal. Calorim. 1995, 45, 417-436. [CrossRef]

14. Szymański, M.; Kozlowska, A.; Malag, A.; Szerling, A. Two-dimensional model of heat flow in broad-area laser diode mounted to a non-ideal heat sink. J. Phys. D Appl. Phys. 2007, 40, 924. [CrossRef]

15. Nakwaski, W. Thermal analysis of the catastrophic mirror damage in laser diodes. J. Appl. Phys. 1985, 57, 2424-2430. [CrossRef]

16. Chen, G.; Tien, C. Facet heating of quantum well lasers. J. Appl. Phys. 1993, 74, 2167-2174. [CrossRef]

17. Smith, W. Mathematical modeling of thermal runaway in semiconductor laser operation. J. Appl. Phys. 2000, 87, 8276-8285. [CrossRef]

18. Menzel, U. Self-consistent calculation of facet heating in asymmetrically coated edge emitting diode lasers. Semicond. Sci. Technol. 1998, 13, 265. [CrossRef]

19. Romo, G.; Smy, T.; Walkey, D.; Reid, B. Modeling facet heating in ridge lasers. Microelectron. Reliab. 2003, 43, 99-110. [CrossRef]

20. Mukherjee, J.; McInerney, J.G. Electrothermal analysis of CW high-power broad-area laser diodes: A comparison between 2-D and 3-D modeling. IEEE J. Sel. Top. Quantum Electron. 2007, 13, 1180-1187. [CrossRef]

21. Szymański, M.; Kozlowska, A.; Malag, A.; Hoser, P. Two-dimensional model of heat flow in edge-emitting laser revisited: A new and more versatile approach. Int. J. Numer. Model. Electron. Netw. Devices Fields 2020, 33, e2745. [CrossRef] 
22. Ziegler, M.; Talalaev, V.; Tomm, J.W.; Elsaesser, T.; Ressel, P.; Sumpf, B.; Erbert, G. Surface recombination and facet heating in high-power diode lasers. Appl. Phys. Lett. 2008, 92, 203506. [CrossRef]

23. Fiore, A.; Rossetti, M.; Alloing, B.; Paranthoen, C.; Chen, J.; Geelhaar, L.; Riechert, H. Carrier diffusion in low-dimensional semiconductors: A comparison of quantum wells, disordered quantum wells, and quantum dots. Phys. Rev. B 2004, 70, 205311. [CrossRef]

24. Boroditsky, M.; Gontijo, I.; Jackson, M.; Vrijen, R.; Yablonovitch, E.; Krauss, T.; Cheng, C.C.; Scherer, A.; Bhat, R.; Krames, M. Surface recombination measurements on III-V candidate materials for nanostructure light-emitting diodes. J. Appl. Phys. 2000, 87, 3497-3504. [CrossRef]

25. Weiss, T.P.; Bissig, B.; Feurer, T.; Carron, R.; Buecheler, S.; Tiwari, A.N. Bulk and surface recombination properties in thin film semiconductors with different surface treatments from time-resolved photoluminescence measurements. Sci. Rep. 2019, 9, 1-13. [CrossRef] [PubMed]

26. Michalewicz, Z.; Fogel, D.B. How to Solve It: Modern Heuristics; Springer Science \& Business Media: New York, NY, USA, 2013.

27. Evans, C.; Jovanovic, V.; Indjin, D.; Ikonic, Z.; Harrison, P. Thermal effects in InGaAs/AlAsSb quantum-cascade lasers. IEE Proc.-Optoelectron. 2006, 153, 287-292. [CrossRef]

28. Hathaway, D.; Shahzad, M.; Sakthivel, T.S.; Suttinger, M.; Go, R.; Sanchez, E.; Seal, S.; Shu, H.; Lyakh, A. Output facet heating mechanism for uncoated high power long wave infrared quantum cascade lasers. AIP Adv. 2020, 10, 085104. [CrossRef]

29. Benson, T.M.; Kendall, P. Variational techniques including effective and weighted index methods. Prog. Electromagn. Res. 1995, $10,1-40$.

30. Steady Temperature in Rectangular Rod (Edge-Emitting Laser). Available online: https://uk.mathworks.com/matlabcentral/ fileexchange/69398-steady-temperature-in-rectangular-rod-edge-emitting-laser?s_tid=srchtitle (accessed on 25 October 2021).

31. Steady Temperature in Rectangular Rod (Different Heat Source). Available online: https://uk.mathworks.com/matlabcentral/ fileexchange/72683-steady-temperature-in-rectangular-rod-different-heat-source?s_tid=srchtitle (accessed on 25 October 2021).

32. Edge-Emitting Laser's Mirror Heating (Surface Recombination). Available online: https://uk.mathworks.com/matlabcentral/ fileexchange/75362-edge-emitting-laser-s-mirror-heating-surface-recombination?s_tid=srchtitle (accessed on 25 October 2021).

33. Thermal Boundary Resistance at Interfaces Between Solids. Available online: https://uk.mathworks.com/matlabcentral/ fileexchange/69224-thermal-boundary-resistance-at-interfaces-between-solids?s_tid=srchtitle (accessed on 25 October 2021).

34. Gęsikowska, E.; Nakwaski, W. An impact of multi-layered structures of modern optoelectronic devices on their thermal properties. Opt. Quantum Electron. 2008, 40, 205-216. [CrossRef]

35. Lee, H.; Chung, K.; Yu, J.; Razeghi, M. Thermal analysis of buried heterostructure quantum cascade lasers for long-wavelength infrared emission using 2D anisotropic heat-dissipation model. Phys. Status Solidi A 2009, 206, 356-362. [CrossRef]

36. Abbas, F.; Pandey, B.J.; Clark, K.; Lascola, K.; Dikmelik, Y.; Robbins, D.; Hinojos, D.; Hodges, K.L.; Roodenko, K.; Gu, Q. Thermal modeling of quantum cascade lasers with 3D anisotropic heat transfer analysis. In Quantum Sensing and Nano Electronics and Photonics XVII; International Society for Optics and Photonics: Bellingham, WA, USA, 2020; Volume 11288, p. 1128808.

37. Szymański, M. Calculation of the cross-plane thermal conductivity of a quantum cascade laser active region. J. Phys. D Appl. Phys. 2011, 44, 085101. [CrossRef] 